\title{
Análise textual dos movimentos sociais no Estado de Pernambuco: um estudo de padrão comportamental
}

\author{
Textual analysis of social movements in the State of Pernambuco: a study of behavioral patterms
}

Análisis textual de los movimientos sociales en el Estado de Pernambuco: un estudio de los patrones de comportamiento

Recebido: 05/10/2021 | Revisado: 12/10/2021 | Aceito: 18/10/2021 | Publicado: 20/10/2021

Edgar Natanael de Vasconcelos Gregório

ORCID: https://orcid.org/0000-0002-1678-8221

Universidade Federal Rural de Pernambuco, Brasil E-mail: edgarnatanael28@hotmail.com

Rosana Alexandre de Sousa

ORCID: https://orcid.org/0000-0002-6242-746X

União de Escolas Superiores da Funeso, Brasil E-mail: rosanasousa1981@gmail.com

\begin{abstract}
Resumo
Os movimentos e manifestações sociais no Brasil são bastante dinâmicos, contudo, os estudos que versam sobre esses eventos nem sempre têm acompanhado esse dinamismo e evolução. Devido à globalização e a rapidez na divulgação da informação, e desinformação, através do advento das comunicações digitais, os movimentos e manifestações sociais no Brasil, em consonância com a América Latina, possuem a mesma tendência de diversificação e complexidade. Não diferentemente, os estudos realizados sobre os movimentos sociais no estado de Pernambuco são frutos de uma conjuntura política bastante heterogênea e que, historicamente, possuem particularidades em virtude de sua conjuntura histórico/social. Diante dessa heterogeneidade novas propostas de análises comportamentais sobre as motivações que permeiam os movimentos sociais no estado de Pernambuco têm por objetivo compreender os comportamentos originários desses movimentos sob a ótica que vai além da hegemonia das ciências sociais, usando Inteligência Artificial. Esta pesquisa científica visa identificar, bem como, analisar os padrões de comportamentos dos movimentos sociais no estado de Pernambuco, obtendo uma compreensão acerca dessas novas configurações da sociedade civil organizada, explicitando os múltiplos tipos de ações preventivas que podem ser tomadas para evitar conflitos e amenizar os riscos. A partir desta compreensão, busca-se explorar as análises de riscos com tendência a confrontos policiais, como também, explorar a diversidade de emprego das tropas policiais militares em movimentos sociais no estado de Pernambuco.
\end{abstract}

Palavras-chave: Movimentos sociais; Inteligência artificial; Sociedade civil; Participação política.

\begin{abstract}
Social movements and manifestations in Brazil are quite dynamic, however, the studies that deal with these events have not always followed this dynamism and evolution. Due to globalization and the speed in the spreading of information, and disinformation, through the advent of digital communications, social movements and manifestations in Brazil, in line with Latin America, have the same trend of diversification and complexity. Not differently, the studies carried out about social movements in the state of Pernambuco are fruits of a very heterogeneous political conjuncture and that, historically, have particularities due to their historical/social conjuncture. In view of this heterogeneity, new proposals of behavioral analysis about the motivations that permeate the social movements in the state of Pernambuco aim at understanding the behaviors originating from these movements from a perspective that goes beyond the hegemony of the social sciences, using Artificial Intelligence. This scientific research aims to identify, as well as, analyze the behavior patterns of social movements in the state of Pernambuco, obtaining an understanding about these new configurations of organized civil society, explaining the multiple types of preventive actions that can be taken to avoid conflicts and mitigate risks. From this understanding, we seek to explore the analysis of risks with a tendency towards police confrontations, as well as, to explore the diversity of employment of military police troops in social movements in the state of Pernambuco.
\end{abstract}

Keywords: Social movements; Artificial intelligence; Civil society; Political participation.

\section{Resumen}

Los movimientos y manifestaciones sociales en Brasil son bastante dinámicos; sin embargo, los estudios sobre estos acontecimientos no siempre han seguido este dinamismo y evolución. Debido a la globalización y a la rápida difusión 
de información y desinformación a través de la llegada de las comunicaciones digitales, los movimientos y manifestaciones sociales en Brasil, en línea con América Latina, tienen la misma tendencia de diversificación y complejidad. No en vano, los estudios realizados sobre los movimientos sociales en el estado de Pernambuco son fruto de una coyuntura política muy heterogénea que históricamente tiene particularidades debido a su contexto histórico/social. Ante esta heterogeneidad, nuevas propuestas de análisis conductual sobre las motivaciones que permean los movimientos sociales en el estado de Pernambuco pretenden comprender los comportamientos originados por estos movimientos desde una perspectiva que supera la hegemonía de las ciencias sociales, utilizando la Inteligencia Artificial. Esta investigación científica tiene como objetivo identificar, así como, analizar los patrones de comportamiento de los movimientos sociales en el Estado de Pernambuco, obteniendo una comprensión sobre estas nuevas configuraciones de la sociedad civil organizada, explicando los múltiples tipos de acciones preventivas que se pueden tomar para evitar los conflictos y mitigar los riesgos. A partir de este entendimiento, buscamos explorar el análisis de los riesgos con tendencia a los enfrentamientos policiales, así como explorar la diversidad del uso de las tropas de la policía militar en los movimientos sociales en el estado de Pernambuco.

Palabras clave: Movimientos sociales; Inteligencia artificial; Sociedad civil; Participación política.

\section{Introdução}

Os estudos sobre os movimentos sociais no estado de Pernambuco são frutos de uma conjuntura que envolve a política, economia, sociologia entre outros. Entretanto, esses movimentos sociais têm mudado de comportamento a cada reformulação política, seja no governo federal, estadual ou municipal (Da Silva Filho, 2010). Esta situação criou condições para novas formas de atuações das forças de segurança do estado, principalmente da Polícia Militar, no qual, realiza estudos de caso, com o objetivo de qualificar e compreender esses novos comportamentos (De Assis, 2014).

Os estudos sobre essas novas mudanças contribuíram para uma contínua convivência entre as diferentes formas da participação das forças de segurança do estado em novos cenários dos movimentos sociais, afilando ainda mais esta relação (Tilly, 2010). O que fundamenta esse novo comportamento da polícia militar nos movimentos sociais é a experiência da corporação em se adequar às diversas formas de manifestações democráticas (Goss \& Prudêncio, 2004).

Diante desses novos comportamentos dos movimentos sociais, foi desenvolvido um script (conjunto de instruções para que uma determinada função seja executada) contendo as principais técnicas de Mineração de Texto - (Text Mining) com o objetivo de analisar as pautas dos movimentos sociais. As pautas de reivindicações são documentos que geralmente antecedem os propósitos dos movimentos sociais (Edileusa et al., 2016). A pauta de reivindicação tem por objetivo expor os anseios de uma determinada categoria representada, contendo no corpo do documento as adaptações, modificações e as inserções de propostas almejadas pela categoria trabalhista, norteando as negociações entre os sindicatos e as empresas (De Paula Leite, 1984).

A aplicação da mineração de texto poderá proporcionar uma análise de risco sobre os documentos estudados com o intuito de subsidiar os tomadores de decisões sobre o comportamento de um determinado movimento, contendo os dados mais relevantes para a compreensão da estrutura comportamental do movimento e uma análise sobre as possíveis probabilidades de intervenção policial.

Desta forma, este trabalho visa propor uma alternativa de analisar tendências de comportamentos, aplicando um dos ramos da inteligência artificial que é a mineração de texto, para que, seja possível uma análise prévia do tipo de ações que deverão ser tomadas pelas forças de segurança do estado de Pernambuco. Esta análise inteligente objetiva diminuir o lapso de complexidade na compreensão da motivação dos movimentos sociais e na aplicação de ações policiais, possibilitando a garantia da democratização de reivindicações pacíficas.

\section{Fundamentação Teórica}

Indivíduos que resguardam e/ou reivindicam demandas relacionadas a causas sociais e políticas, geralmente, formam o que convencionamos de movimentos e manifestações sociais. É uma maneira encontrada pela população de se organizar, de 
expressar os seus anseios e de expressarem o que julgam ser direitos. São fenômenos históricos, frutos de demandas sociais, que vão modificando e influenciando mudanças sociais estruturais (Nunes, 2014).

\subsection{Movimentos sociais contemporâneos}

Os movimentos sociais podem ser divididos em dois tipos: Conjuntural e Estrutural (Corrêa \& De Almeida, 2012). O movimento social denominado de conjuntural tem curta duração, segue demandas momentâneas (a exemplo das reivindicações pelo não aumento do preço das passagens), entretanto o movimento denominado de estrutural possui reivindicações e aspirações a longo prazo, baseado em causas mais culturalmente enraizadas, como podemos citar os movimentos explanam questões como racismo ou Reforma Agrária.

Existem movimentos que buscam discordar ou apoiar causas e/ ou ideologias afirmadas pelos governos em exercício, a depender das demandas por esses levantadas também representam formas de movimentos e manifestações sociais. Porém, preciso fazer a diferenciação de movimento social e de manifestação espontânea (Marques, 2013). Sendo assim, manifestações espontâneas acontecem sem que necessariamente necessitem de ideais em comum (Marques, 2013).

As reivindicações por reconhecimento, visibilidade e por direitos civis, fez surgir o que chamamos de movimentos sociais contemporâneos (Paiva, 2018). Em evidência nos últimos tempos, o movimento LGBTQIA+ (sigla que significa: Lésbicas, Gays, Bissexuais, Travestis, Transexuais, Transgêneros, Queer, Intersexo e Assexual e mais), outrora conhecido com GLS, LGBT, atua mundialmente buscando igualdade social e de direitos e contra o preconceito (Tanizaka, 2020). A Parada do orgulho LGBTQIA+ de São Paulo, que acontece anualmente na Avenida Paulista e atrai turistas de todo o mundo, é um exemplo da busca por visibilidade de seus integrantes e apoiadores. Assim como em diversos lugares do mundo, o objetivo principal da Parada é a luta contra a LGBTfobia.

Movimentos contra o racismo, embora não sejam o que podemos citar de atuais, ainda se fazem bastantes presentes nas sociedades contemporâneas. Reivindicações pelo fim do Racismo Estrutural, pelo acesso à igualdade de oportunidades, acessam a espaços que não se fazem comuns a todos os brasileiros e pelo fim da marginalização em virtude da raça são bandeiras levantadas pelos defensores desses movimentos (Paiva, 2018). Movimentos estudantis foram muito atuantes no Brasil na década de 60, a exemplo da "Marcha dos Cem Mil". Reivindicações por ensino público de qualidade, maiores dispêndios de verbas públicas são causas presentes entre os estudantes. Eles possuíam e ainda possuem várias organizações representativas, a exemplo da UNE (União Nacional dos Estudantes) e da UEE (Uniões Estaduais dos Estudantes). Esses movimentos se fizeram presentes nas manifestações dos anos 90, Diretas Já, em oposição ao governo do ex-presidente Fernando Collor de Melo, bem como mais recentemente na mobilização estudantil de 2016 (Lacerda, 2017).

O movimento feminista ou feminismo é um movimento nascido no Séc. XIX e que perdura até os dias atuais, liderado por mulheres que realizaram campanhas políticas inicialmente pautadas no direito ao sufrágio universal, liberdades civis e autonomia legal, como direitos trabalhistas e ao divórcio. Entretanto, ao longo das décadas, as reivindicações feministas foram se pautando em questões como direitos reprodutivos, violência doméstica, licença maternidade, igualdade de remuneração, assédio e violência sexual. As reivindicações feministas são variáveis entre nações e comunidades.

No Ocidente, historicamente, o movimento feminista, passou por denominadas, três ondas de atuação, sendo a primeira mais pautada em causas que envolviam mulheres brancas de classe média ou alta (sufrágio das mulheres e igualdade política). A segunda onda buscou combater desigualdades sociais e culturais, arrebatando também mulheres negras e pardas, além de mulheres estrangeiras pertencentes a países em desenvolvimento que buscavam solidariedade. Esta segunda onda também se relaciona a causas que envolviam questões de sexualidade. A terceira onda do feminismo perpetra a abordagem às desigualdades financeiras sofridas por mulheres em igualdade de cargos com homens, às questões sociais e culturais e inclui a necessidade de maior influência das mulheres na política e na mídia. Nesta terceira onda as feministas também tiveram que 
manter o foco nos direitos reprodutivos das mulheres, como o direito ao aborto. Também vislumbraram questões de interseccionalidade, como opressão por questões sociais, de raça e de orientação sexual. Há os que ainda definem a existência da quarta onda do Feminismo, nascida pelos anos de 2010, buscando representatividade e que analisa sistemas interligados de poder que contribuem para a estratificação de grupos tradicionalmente marginalizados (De Melo, 2018).

Outro movimento a ser referenciado neste trabalho é o movimento Black Bloc surgido na década de 80, na Alemanha Ocidental e que ganhou notoriedade no Brasil após as manifestações de junho de 2013. As manifestações que ocorreram no Brasil a partir de maio/junho de 2013 contra o aumento da tarifa do transporte organizadas pelo Movimento Passe Livre (MPL), trouxeram um debate novo na imprensa brasileira: o black bloc (Pinto, 2017).

\subsection{Tecnologia como aliada: Os movimentos sociais em rede}

A cultura do debate em rede nasce com o advento e proliferação da internet. Desde as manifestações de 2013 que estes debates se fazem presentes nas redes sociais, especialmente no Facebook, Twitter e Whatsapp. Os usuários das redes se organizam - pontos de encontro, horários, vestimenta, entre outros - e repassam as informações de forma instantânea, atingindo um número expressivo de pessoas (Pinto, 2017). Mais recentemente, debates políticos que envolvem as ditas "fake news" têm se intensificado nas redes sociais como formas também de organização popular a fim de combater interferências desproporcionam e enganosas que venham a alterar os cenários políticos de algumas nações.

A falta de fronteiras físicas superdimensiona o alcance da internet e, consequentemente, da globalização. Podemos citar a existência de movimentos transnacionais, como o Fórum Social Mundial, evento que é organizado por diferentes movimentos sociais pelo mundo e tem o objetivo de apresentar soluções para problemas contemporâneos de transformação social global neoliberal, em contraponto ao Fórum Econômico Mundial (De Santana Batalha \& Arturi, 2006).

\subsection{Mineração de texto (Text mining)}

A mineração de texto (Text Mining) tem por objetivo interpretar a linguagem natural dos documentos de texto, sendo capaz de compreender sua imprecisão e incerteza. A mineração de textos envolve diversas áreas da computação, como mineração de dados (Data Mining), aprendizado de máquina (Machine Learning), recuperação de informação, estatística, linguagem computacional, entre outros, com o intuito de transformar o texto analisado em alguma coisa interpretado por um computador (Machado et al., 2010).

O principal objetivo da mineração de texto é encontrar termos consideráveis em documentos com grande volume de dados para estabelecer padrões de relacionamentos entre eles e um grau de sentimento com base na regularidade e temática dos termos encontrados (Serapião et al., 2010).

Segundo Calixto (2013), às diversas etapas aplicáveis à mineração de textos podem-se considerar quatro delas que se destacam, como: coleta de documentos, pré-processamento, extração de conhecimento e avaliação e interpretação dos resultados. Inicialmente a coleta de documentos objetiva realizar algum tipo de relacionamento com o tipo de conhecimento que se deseja obter. A técnica utilizada para esta primeira etapa pode ser tanto o processamento de linguagem natural como também a recuperação de informação (Calixto, 2013).

A peculiaridade desta primeira etapa é o aprendizado de forma não supervisionada, ou seja, o especialista não interfere no agrupamento e na análise de sentimento do texto analisado, desta maneira, as informações são extraídas sem qualquer interferência do analista, seja ela, de cunho sentimental ou intencional, facilitando desta forma um resultado imparcial e imprevisível das informações coletadas e preparando o resultado para as próximas etapas (Corrêa et al., 2012).

Após a primeira etapa aplicada ao documento em questão, o texto é estruturado de forma padronizada, contudo, essa estrutura mantém todas as características naturais do texto analisado, desta forma, as demais etapas poderão manipular e 
analisar o mesmo texto. No final desta primeira etapa terá como resultado uma estrutura contendo atributo e valor sendo representada em uma tabela (Corrêa et al., 2012).

Na segunda etapa são definidos os termos que serão utilizados para a extração dos dados e para isto, são eliminados inicialmente os stopwords (palavras irrelevantes), ou seja, termos insignificantes para a análise de texto. Essas palavras irrelevantes podem ser artigos, advérbios e pronomes. Outras análises são aplicadas como stemming e thesaurus, que tem por objetivo identificar variações morfológicas e sinônimas dos termos, a fim de reduzir o conjunto da população textual. (Corrêa et al., 2012).

Contudo, na terceira etapa, a extração do conhecimento é aplicada utilizando o algoritmo de extração automática de conhecimento buscando informações desconhecidas e imperceptíveis no documento até o momento, mas que possam ser importantes. A utilização do algoritmo de extração textual busca realizar o agrupamento de objetos similares aplicando medidas de proximidade entre as palavras e, ao mesmo tempo, busca agrupar palavras com características heterogêneas. Por fim, na quarta e última etapa é utilizada a avaliação e interpretação dos resultados, onde se utiliza o papel do analista para avaliar se os resultados extraídos são satisfatórios, e em caso negativo, que etapas poderiam ser refeitas para melhorá-los (Berry \& Kogan, 2010).

\subsection{Padrão comportamental}

Segundo a Psicologia o "comportamento" é estudado de forma extensa ou abrangente onde depende de uma ampla escala de fatores, dentre os quais podem ser destacados: as diferentes atividades praticadas que são diretamente observáveis e que podem ser registráveis, os processos fisiológicos e os processos cognitivos (Larrañaga Uriarte, 2008).

Para Logen (1997) o comportamento pode ser relacionado com processos de etapas, onde um indivíduo percorre para dar respostas a uma determinada situação, procurando satisfazer uma determinada necessidade. No entanto um padrão pode ser explicado como algo ou comportamento que se repete, ou seja, que possui uma regra, uma lógica ou uma conformação bem definida, ou seja, segundo o padrão comportamental é uma maneira de agir ou de ação que se repete diante de situações similares (Jesus, 2013). Destarte, Le Bon (1895) afirma que grupos organizados podem desempenhar um papel comportamental semelhante, agitando no inconsciente coletivo através do anonimato, contágio e a sugestionabilidade (LE BON, 1895, p. 3)

\section{Análise de Padrão Comportamental dos Movimentos Sociais no Estado de Pernambuco}

Por cumprir os requisitos necessários para a execução da mineração de texto, foi utilizada a ferramenta Rstudio na versão 3.4.2 para executar o script desenvolvido, como mostrado na Figura 1. O Rstudio é um Ambiente de Desenvolvimento Integrado - IDE, para a linguagem de programação R (Allaire, 2012). A linguagem de programação R é destinada a programação estatística e análise de dados e pode ser executada em diferentes sistemas operacionais, como Windows, Mac OS e Linux (Reisen \& Silva, 2011).

Uma das características destacáveis da linguagem de programação R é a aplicação de Machine Learning (Aprendizado de Máquina). Diversos algoritmos de Machine Learning podem ser desenvolvidos através desta linguagem, como: Redes Neurais, Deep Learning, Naives Bayes, Regressão Linear, Text Mining, entre outros (Herold, 2019). 
Figura 1 - IDE RStudio.

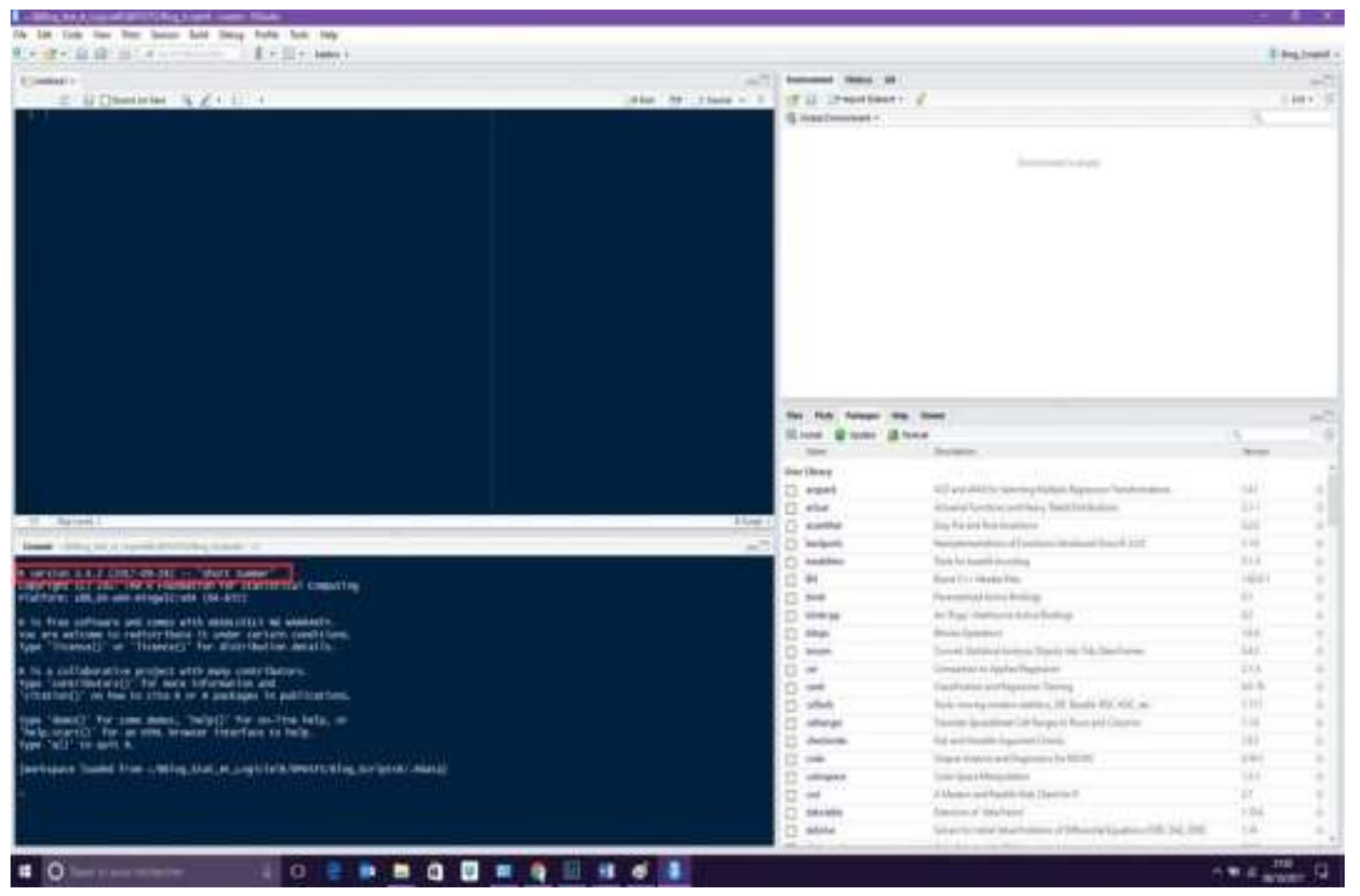

Fonte: Autores.

\subsection{Coletas de dados}

A presente subseção expõe os procedimentos metodológicos utilizados na pesquisa, detalhando o delineamento e método usados, com foco na seleção dos casos, coleta e análise dos dados.

\subsubsection{Delineamento da pesquisa}

O delineamento desta pesquisa se dá pela pesquisa quantitativa.

\subsubsection{Pesquisa quantitativa}

Na pesquisa quantitativa as variáveis que serão observadas são mínimas, diretas e principalmente medidas em escalas numéricas (Wainer, 2007). A utilização da pesquisa quantitativa tem como objetivo assumir que as variáveis selecionadas pelo pesquisador podem ser medidas de forma objetiva (Da Silva et al, 2014).

Genericamente, a pesquisa quantitativa explica a ocorrência de um determinado fenômeno e para isso são utilizados métodos que ancorados na representação de uma realidade temporal observada. Isso indica que a pesquisa quantitativa permitirá obter números os quais será possível realizar a aplicação da estatística com o intuito de validar a proposta da pesquisa (Manzato \& Santos, 2012).

\subsubsection{Técnicas de análise de dados}

As técnicas utilizadas para a realização de análise de dados serão aplicadas para a obtenção e compreensão dos dados adquiridos utilizando um conjunto de métodos para a interpretação dos resultados. Diante do contexto, Martins e Theóplilo (2007 p.69), afirmam que a análise de dados consiste em esquadrinhar, classificar e categorizar os dados coletados.

No entanto, Merriam (1998) afirma que uma análise de dados inseridos a um estudo de pesquisa qualitativa é classificada de complexa e deve ter o seu início ainda no campo de pesquisa, através das observações, reflexões e das anotações sobre o ambiente em que estão inseridos. 
Na percepção de Bardin (2004) a análise de dados pode ser dividida em 03 (três) etapas: sendo a primeira Pré-análise, a segunda Exploração do material e a terceira o tratamento dos resultados. A pré-análise é a fase operacional para organizar as ideias e os materiais obtidos e que será avaliado, formulando as proposições e consequentemente os objetivos que irão fundamentar o resultado final da análise.

Para o desenvolvimento e aplicações das análises, realizou-se uma investigação nas publicações dos principais jornais eletrônicos, como também, em páginas eletrônicas oficiais de sindicatos mais atuantes no estado de Pernambuco, com objetivo de catalogar diversas pautas de reivindicações dos movimentos sociais a partir da data de 01 de janeiro de 2020 até a data de 30 de junho de 2021, totalizando 384 registros de pautas reivindicatórias dos movimentos sociais, ilustrado na Tabela 1.

Tabela 1 - Coletas de Dados.

\begin{tabular}{|l|c}
\hline 01 de janeiro de 2020 a 31 de dezembro de 2020 & 177 \\
\hline 01 de janeiro de 2021 a 30 de junho de 2021 & 207 \\
\hline Total dos registros & 384 \\
\hline
\end{tabular}

Fonte: Autores.

Após as coletas das pautas reivindicatórias, os dados foram plotados e organizados no formato de planilha eletrônica (Microsoft Excel), com o objetivo de visualizar os dados de forma sequencial, separando, qualificando e registrando os dados mais relevantes para a análise em quatros variáveis, como: a data da manifestação social, o horário, o destino final e a motivação do movimento, como exemplificado na Tabela 2.

Tabela 2 - Exemplo de registro dos dados.

\begin{tabular}{l|c|l|l}
\hline Data do movimento & Horário & Destino final & Motivação \\
\hline Data a & Horário a & Rua a & Motivação a \\
\hline Data b & Horário b & Rua b & Motivação b \\
\hline Data c & Horário c & Rua c & Motivação c \\
\hline
\end{tabular}

Fonte: Autores.

Com o objetivo de preservar a identidade e os direitos autorais dos movimentos reivindicatórios analisados, foram ocultados desta pesquisa os nomes e endereços eletrônicos dos sindicatos analisados, como também, dos jornais digitais pesquisados.

\subsection{Definição dos experimentos}

A execução dos experimentos foi realizada da forma que se segue. O primeiro experimento consiste em analisar a variável "Motivação do movimento social". A variável "Motivação do movimento social" contém todas as motivações reivindicatórias dos movimentos sociais dentro do estado de Pernambuco durante a pesquisa realizada, sendo essas motivações informadas pelo próprio grupo de reivindicadores ou seus representantes.

A análise sobre a motivação dos movimentos sociais é para compreender quais as motivações que mais se reivindicam dentro do estado de Pernambuco durante o período pesquisado, destacando esses motivos através de uma representação visual, dando um maior destaque às palavras que aparecem com uma maior frequência através de uma nuvem de palavras.

$\mathrm{O}$ segundo experimento consiste em analisar três registros de um único sindicato postado em sua rede social em três períodos diferentes. A variável analisada será também a "Motivação do movimento social”, contudo, o objetivo é identificar os 
sentimentos textuais das pautas publicadas. Análise textual ou de sentimento pode ser entendido como o processo de determinar se o sentimento do texto é positivo, negativo ou neutro (Vilela et al., 2020). As análises de sentimentos utilizam uma combinação de processamento de linguagem natural - PNL, como também, as técnicas de aprendizado de máquinas Machine Learning (De Souza Prais \& Da Rosa, 2017).

\subsubsection{Experimento 1: Execução e avaliação}

As nuvens de palavras são representações visuais de dados de textos, onde normalmente são usadas para descrever metadados de palavras-chave. As palavras-chaves podem ser palavras solteiras (isoladas) e a sua importância dentro do texto é mostrada através da frequência em que essa palavra se repete aumentando o tamanho e a cor da fonte. (Carvalho et al., 2012).

Segundo Vilela et al. (2020), os três pilares da nuvem de palavras são recursos navegacionais, método heurístico de análise e apresentação e visualização dos dados. O recurso navegacional consiste em facilitar o acesso ao texto ou trecho do texto através do clique em palavras da nuvem, considerando a eficácia intuitiva de qualquer volume de textos.

Contudo, o método heurístico de análise verifica o motivo pelo qual uma determinada palavra se repete dentro do texto, estudando a sua incidência. Diante disso, a nuvem de palavras possui um grande potencial para resolver problemas até antes ocultados nos textos, como também, a possibilidade de responder uma questão de pesquisa, mas propensa a um determinado caminho. Por fim, a apresentação e a visualização de dados são apresentadas de forma resumida e direta revelando imediatamente as palavras-chave mais comuns em um determinado documento (De Souza Prais \& Da Rosa, 2017).

Antes da aplicação e construção da nuvem de palavras, alguns processos de limpeza dos dados foram realizados nos registros. Inicialmente foi criado um conjunto de palavras para serem retiradas do texto, conhecidos como stopwords (palavras irrelevantes), obtendo assim um novo texto, e por fim foi criada uma matriz de palavras (tdm) para ser construída a nuvem de palavras. Os comandos utilizados para a extração das palavras irrelevantes e a criação da matriz de palavras são mostrados na Figura 2.

Figura 2 - Extração de Palavras Irrelevantes.

1 stopwords_pt <- c(stopwords("pt"), "é", "sr", "sra", "todos", "para", "sua", "nenhum")

2 tdm $<$ - data.frame $($ word $=$ stopwords_pt)

Fonte: Autores.

Para a criação da nuvem de palavras é necessária a instalação do pacote "wordcloud" no RStudio, linha 1, como também, o pacote "RColorBrewer", linha 2, responsável de colorir as palavras com mais frequência na nuvem de palavras, com o objetivo de uma visualização mais facilitada para os usuários finais, conforme mostrado na Figura 3. 
Figura 3 - Script para a criação da nuvem de palavras.

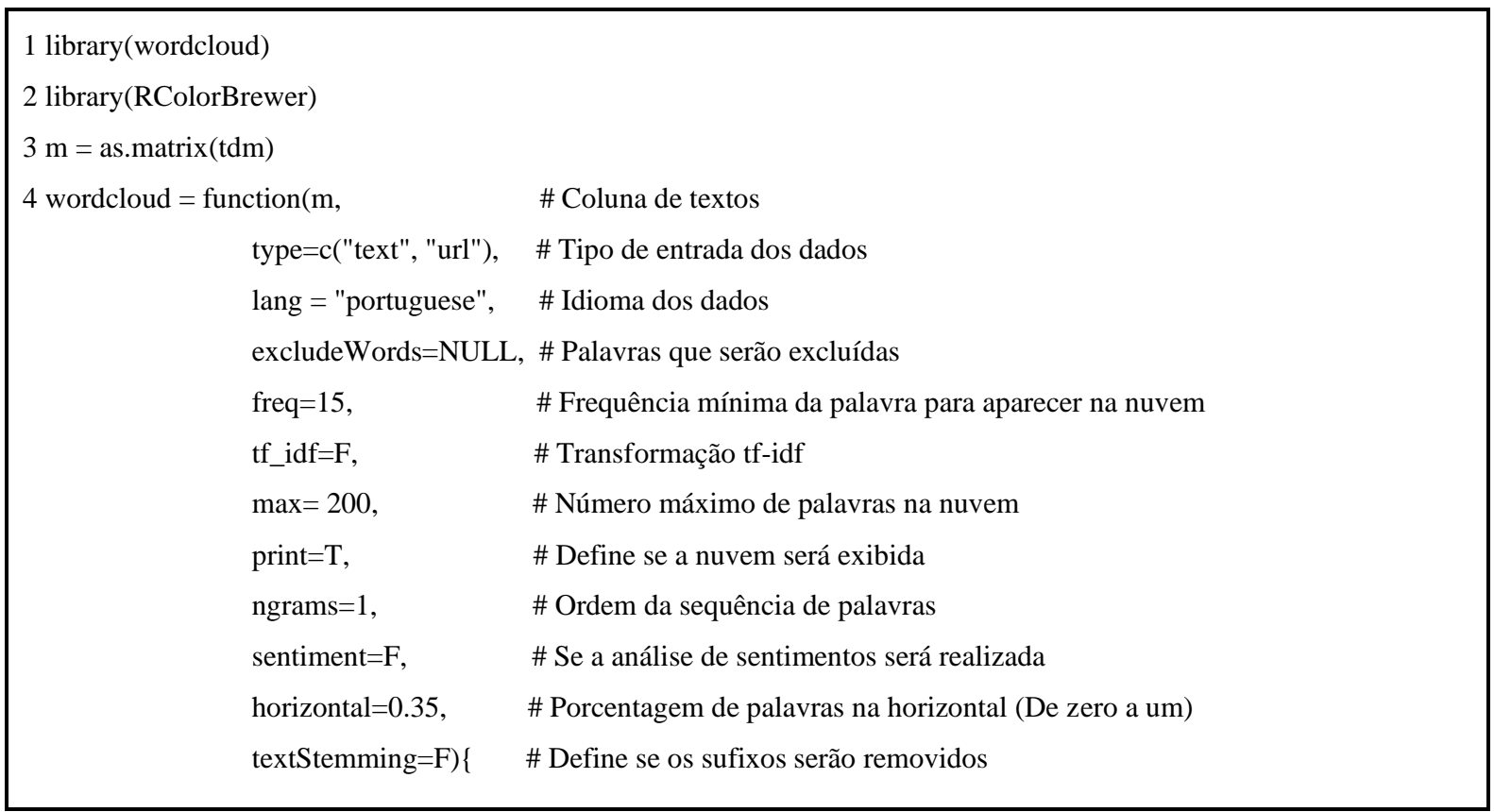

Fonte: Autores.

Na linha 4 a função "wordcloud" tem por objetivo receber os parâmetros para a criação da nuvem de palavras. Diante dos parâmetros, pode-se destacar o "freq" - onde determina a frequência mínima em que uma palavra deve conter para ser registrada na nuvem de palavras (Figura 4).

Figura 4 - Nuvem de palavras.

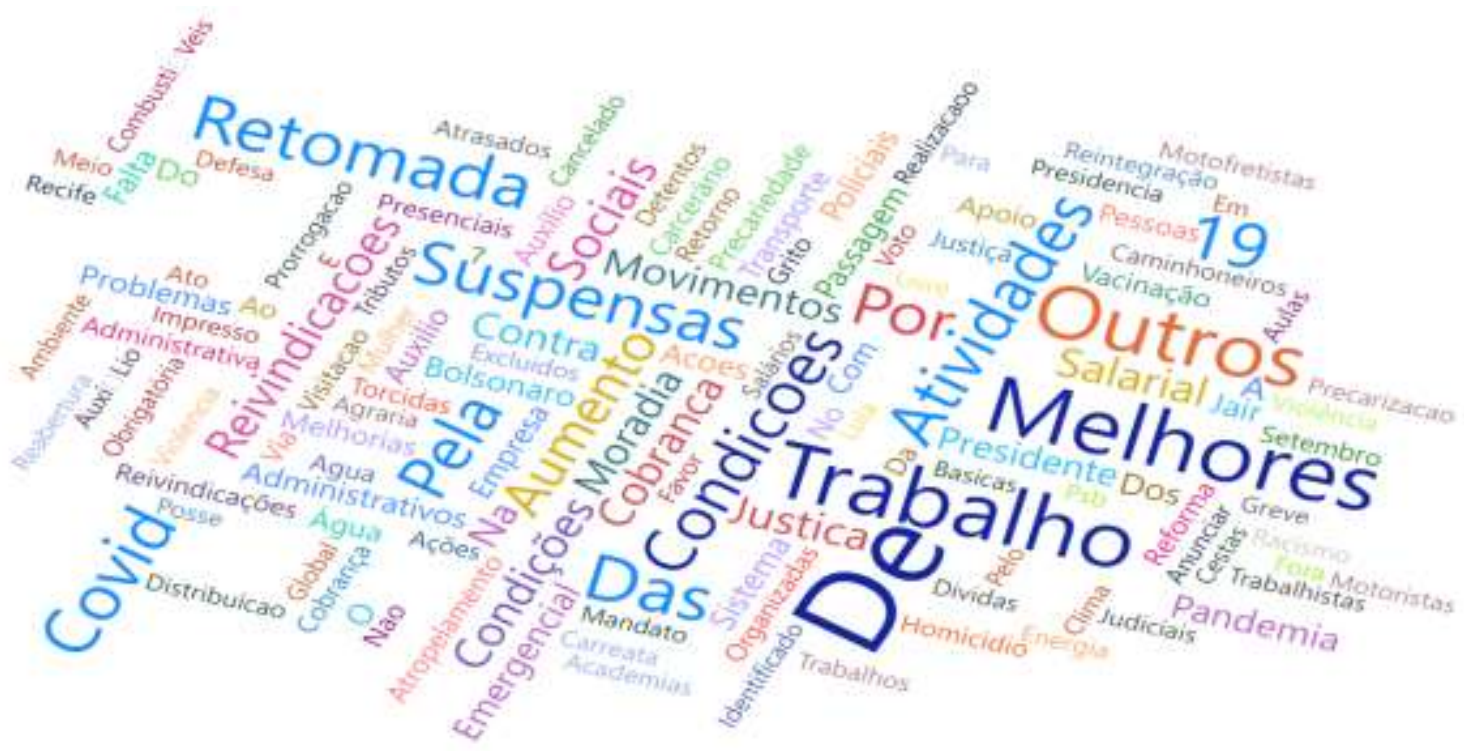

Fonte: Autores.

É possível identificar na nuvem de palavras os fragmentos de textos mais frequentes pelo seu maior tamanho e cores variadas. Pode-se observar que as palavras que mais se destacam são: "Trabalho”, "Melhores”, "Condições”, e possuem a cor azul escuro. No entanto, o segundo grupo de palavras mais frequentes são: "Retomada", "Suspensa”, "Atividades" e "Covid19", destacadas de cor azul claro. 
Desta forma é possível compreender que os movimentos sociais ocorridos de 01 de janeiro de 2020 a 31 de dezembro de 2020 até 01 de janeiro de 2021 a 30 de junho de 2021, possuem uma tendência de fortes motivações relacionadas aos problemas socioeconômicos causados pelo vírus da Covid-19. Diante dessa informação pressupõe-se que os atos reivindicatórios a partir do ano pesquisado possuem um caráter não violento a própria sociedade civil, como também, a estruturação organizada do governo estadual, pressupondo que, as atuações das forças de segurança do estado sejam empregadas de forma a orientar os manifestantes na condução do protesto e assegurar ao direito de protesto.

\subsubsection{Experimento 2: Execução e avaliação}

O Text Mining ou análise de texto é responsável pelo processamento, transcrição e compreensão da linguagem humana ajudando a entender, classificar e analisar os textos. As técnicas aplicadas utilizam processos linguísticos, estatísticos e de aprendizado de máquina (Machine Learning), que modelam e estruturam todo o conteúdo dos textos.

Diante das diversas técnicas aplicáveis na mineração de texto, esta pesquisa faz o uso de três técnicas, sendo a primeira Detecção de Intenção, o segundo Análise Sentimental e por fim, o Processamento de Ações. A detecção de intenção é um procedimento utilizado no processamento de linguagem, no qual, permite determinar aproximadamente qual a finalidade de cada palavra, realizando assim um agrupamento dessas palavras com o objetivo de extrair a intenção da escrita do orador.

Contudo, a análise sentimental pode ser entendida como a técnica utilizada para determinar possíveis tons emocionais em um agrupamento de palavras, com o objetivo de tentar compreender quais as atitudes, opiniões e emoções expressas nas palavras e, desta forma, após a identificação dos termos, como também, as intenções e os tons de cada grupo de palavras, o processamento de ações é responsável por identificar quais os procedimentos possíveis a serem realizáveis.

Inicialmente foram analisadas as três pautas de eventos em três datas distintas, sendo a primeira pauta na data 14/01/2021, o segundo na data 15/01/2021 e o terceiro e último na data 18/01/2021. O segundo experimento foi analisado em três etapas, sendo que a primeira foi à criação e a plotação dos dados em gráficos, com o objetivo de identificar os fragmentos dos textos que mais se destacam dos demais, realizando uma melhor compreensão das pautas do evento.

O primeiro gráfico refere-se aos termos mais utilizados na pauta da data 14/01/2021. Como mostrado na Figura 5, pode-se destacar as palavras mais utilizadas no ato reivindicatório nesta data, como: Companheiro. De forma seqüenciada, a palavra que mais se destaca com mais frequência é em primeiro lugar "Companheiro", porém, mais 07 (sete) palavras se destacam com a mesma freqüência no texto avaliado, são eles: Assembléia, X, Cisneiro, Favor, Liberdade, Reintegração e Sindical. 
Figura 5 - Gráfico de Frequência - 14/01/2021.

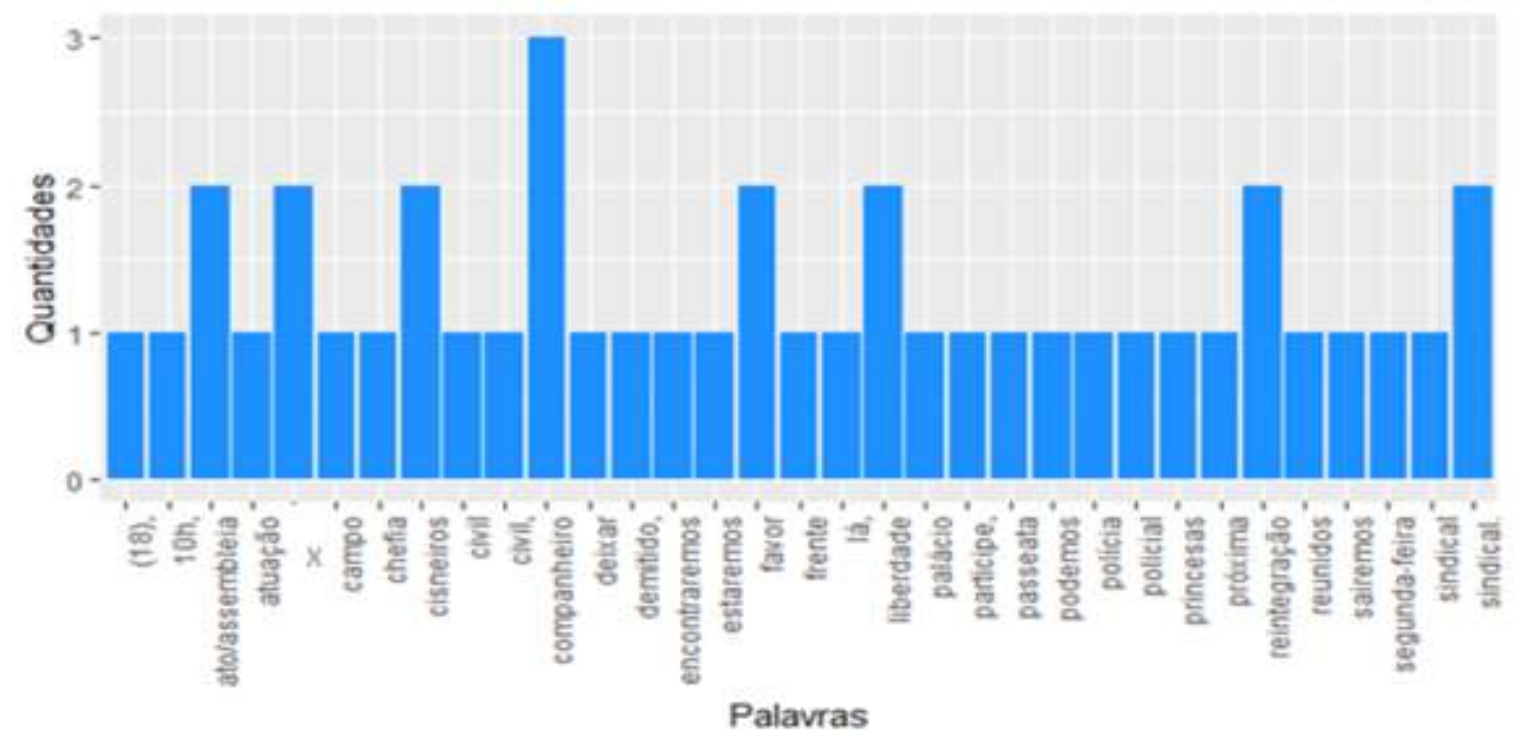

Fonte: Autores.

O segundo gráfico refere-se à aos termos mais utilizados na pauta da data 15/01/2021, contudo, não houve uma palavra que se destacam das demais, no entanto, as palavras de mais frequência foram: Ato, X, Cisneiro, Civil, Policia e Readmissão, como mostrado na Figura 6.

Figura 6 - Gráfico de Frequência - 15/01/2021.

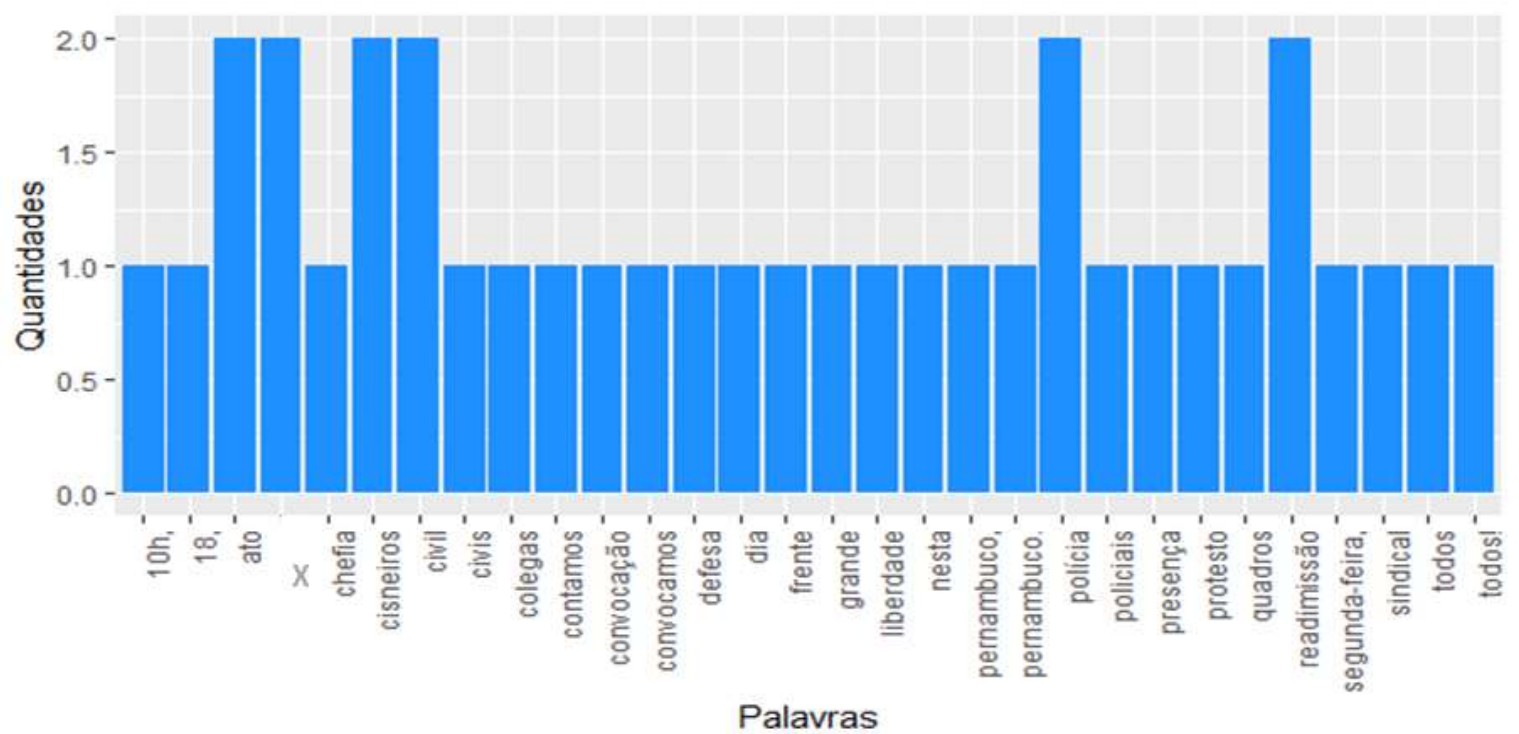

Fonte: Autores.

O terceiro e último gráfico refere-se aos termos mais utilizados na pauta da data 18/01/2021, no entanto, não houve uma palavra com maior destaque, contudo, as palavras de mais frequência foram: Ato, X, Cisneiros, Civil, Policia e Readmissão, como mostrado na Figura 7. 
Figura 7 - Gráfico de Frequência - 18/01/2021.

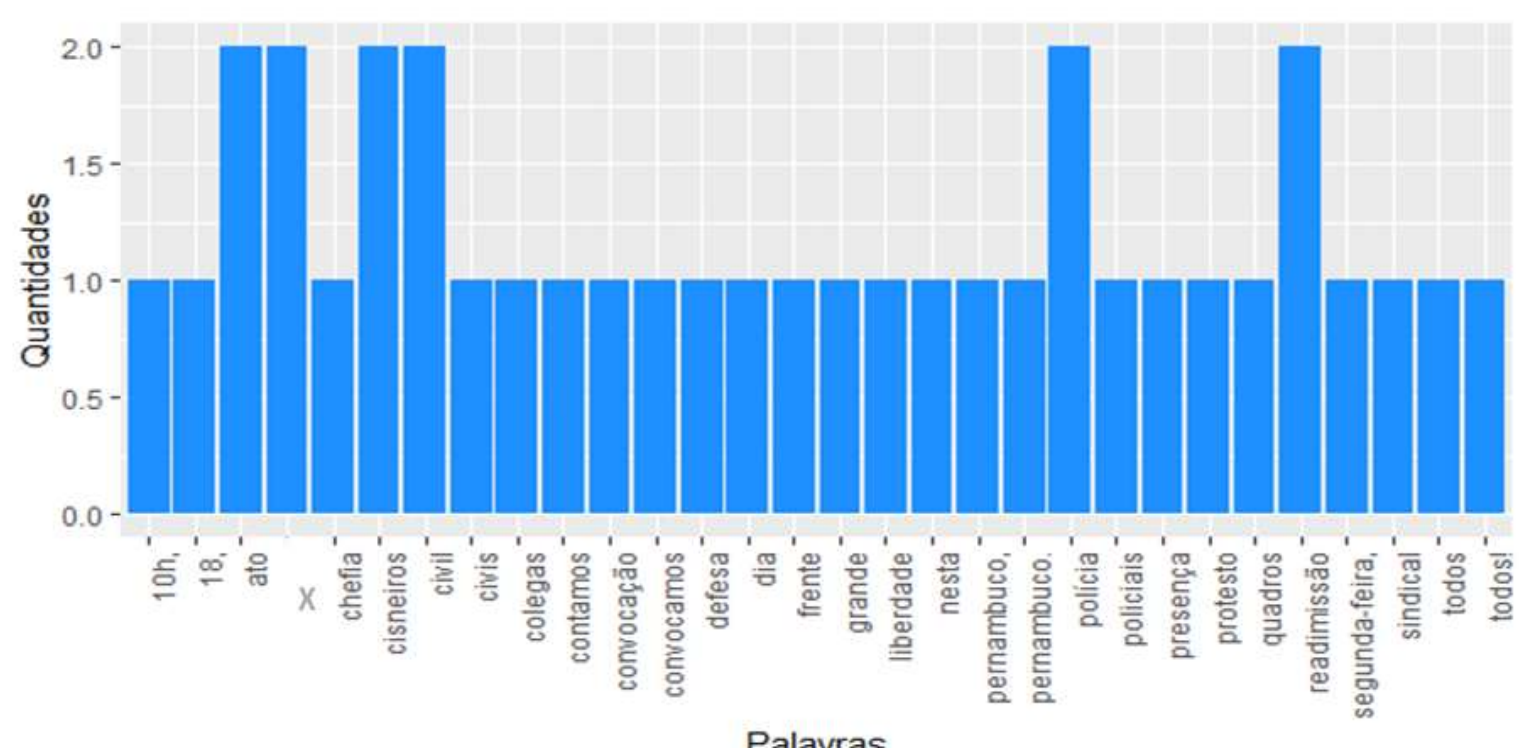

Fonte: Autores.

A segunda etapa do segundo experimento consiste na correlação linear entre as características das palavras utilizadas nas pautas dos eventos, indicando assim, uma forte relação entre a pauta publicada no dia 14/01/2021 e a pauta publicada no dia 15/01/2021, revelando uma forte tendência na coesão dos textos e consequentemente nos objetivos dos protestos. Contudo, temos uma propensão de baixa correlação entre a pauta na data 15/01/2021 e a pauta na data 18/01/2021, como também entre a pauta da data 18/01/2021 com a pauta do dia 14/01/2021, como mostra a Figura 8. Pode-se compreender que, a intenção da pauta do dia 14/01/2021 e do dia 15/01/2021 estão coesos, contudo, na pauta do dia 18/01/2021 a coesão textual diminui, ou seja, os objetivos e intenções textuais são modificados de forma direta.

Figura 8 - Correlação Linear.

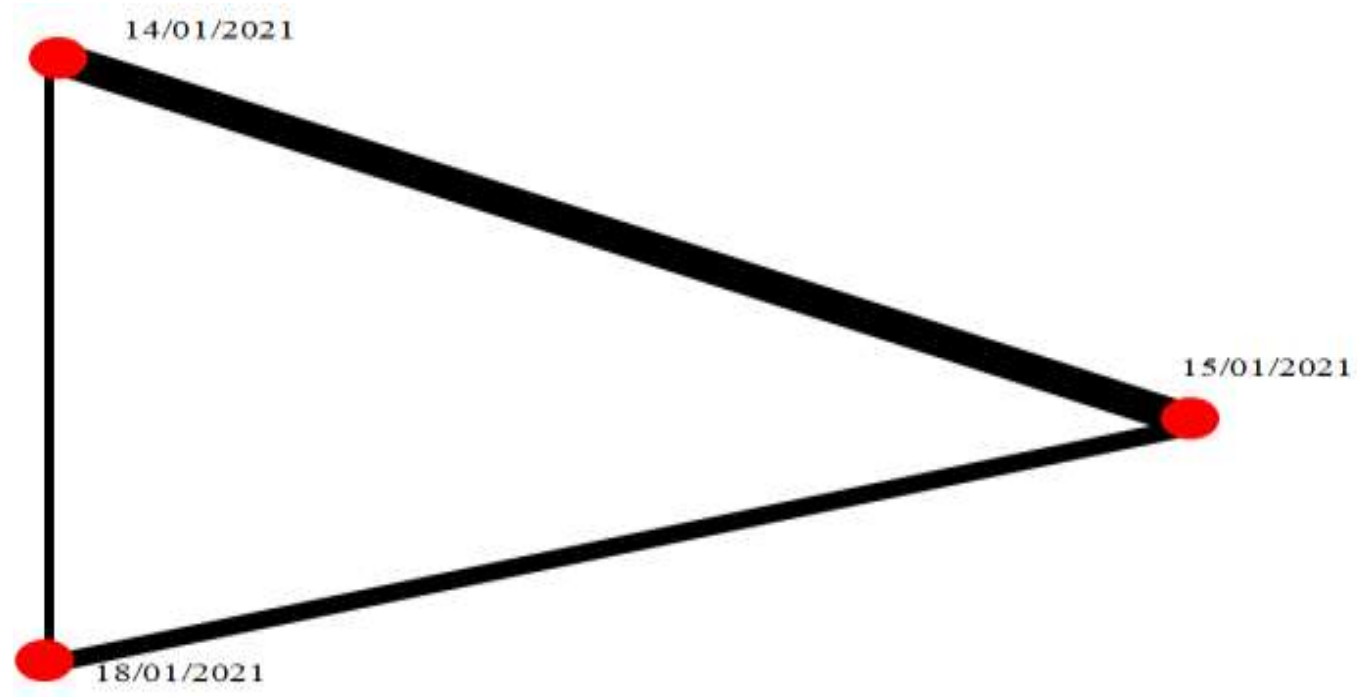

Fonte: Autores.

A terceira etapa consiste em realizar uma análise de sentimento das três pautas em questão, onde possuem uma clara tendência de sentimentos negativos em relação ao governo do estado de Pernambuco (Figura 9). Na data 14/01/2021 a pauta 
possui uma tendência negativa em relação ao sentimento em relação ao governo do estado, todavia, na data 15/01/2021 o sentimento negativo perde força e, por fim, na data 18/01/2021 ocorre um decréscimo significativo no sentimento negativo em relação ao governo estadual, confirmando a mudança de postura analisada na Figura 8.

Figura 9 - Análise de Sentimento.

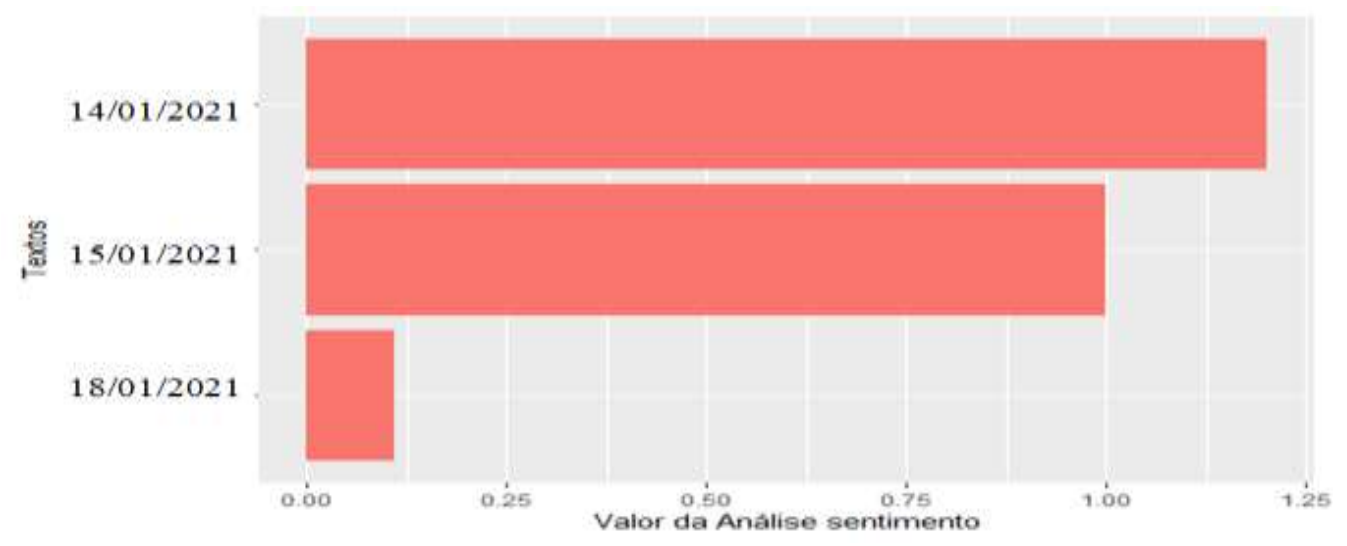

Fonte: Autores.

\section{Considerações Finais}

Ante o exposto, pode-se concluir que os movimentos sociais no estado de Pernambuco são um marco histórico presente na história do Brasil, em relação às conquistas e lutas pelos direitos sociais e políticos. A importância dos movimentos sociais é que colaboram efetivamente para o processo democrático no estado pernambucano e do país como o todo e principalmente para a concretização dos direitos reivindicatórios e humanos.

Os resultados desta pesquisa imparcial apontam que a realização de uma análise de dados permite obter um panorama geral do movimento reivindicatório e da sua motivação revelando riquezas de detalhes da sua construção, motivação e histórico. É por meio dessa análise que é possível desenvolver estratégias reais de atuação das forças de segurança nesses movimentos.

A aplicação da inteligência artificial para análise de padrões comportamentais dos movimentos sociais dentro do estado de Pernambuco visando tomadas de decisões ainda mais técnicas e consistentes, garantindo a efetividade do emprego das forças de segurança de forma estratégica, observando quais pontos precisam ser mais bem monitorados, buscando respostas cada vez mais eficientes e eficazes para a causa pública. Portanto, o conhecimento prévio dos modus operandi dos movimentos sociais pelas forças de segurança poderá proporcionar ações pacíficas que atenderão às expectativas dos protestantes o que ocasionará no impedimento de um conflito entre as partes.

\section{Referências}

Allaire, J. (2012). RStudio: integrated development environment for R. 770(394), 165-171.

Bardin, L. (2008). Análise de conteúdo (4 a edição, pp. 70). Edições.

Berry, M. W., \& Kogan, J. (Eds.). (2010). Text mining: applications and theory. John Wiley \& Sons.

Calixto, I. C. A. C. (2013). Towards the generation of a database for scientific research in Natural Language Processing with an Information Extraction system (Doctoral dissertation).

Carvalho Jr, P. M., Rosa, R. S. L., Sgambatti, M. S., Adachi, E. A., \& Carvalho, V. C. (2012). Avaliação do programa de residência multiprofissional em saúde da família: uma análise qualitativa através de duas técnicas. Revista HUPE, 11(1), 114-9.

Corrêa, F., \& de Almeida, M. A. B. (2012). Teorias dos movimentos sociais e Psicologia Política. Revista Psicologia Política, 12(25), 549-570. 
Corrêa, G. N., Marcacini, R. M., \& Rezende, S. O. (2012). Uso da mineração de textos na análise exploratória de artigos científicos.

Da Silva, D., Lopes, E. L., \& Junior, S. S. B. (2014). Pesquisa quantitativa: elementos, paradigmas e definições. Revista de Gestão e Secretariado, 5(1), 01-18.

Da Silva Filho, N. G. (2010). O embate entre os movimentos sociais e o estado: a história de Pernambuco durante o Regime Militar (1964-1966). X Encontro Nacional de História Oral. Testemunhos: história e política (sp). Universidade Federal de Pernambuco. Centro de Filosofia e Ciências Humanas. Disponível na http://www. encontro2010. historiaoral. org. br.

De Assis, M. E. A. (2014). O Museu da Abolição-MAB e os movimentos sociais de Pernambuco. Revista Memória em Rede, 6(11).

De Melo, H. P., \& Thomé, D. (2018). Mulheres e poder: histórias, ideias e indicadores. Editora FGV.

De Paula Leite, M. (1984). Reivindicações sociais dos metalúrgicos. Centro de Estudos de Cultura Contemporânea.

De Santana Batalha, E., \& Arturi, C. S. (2006). Movimentos Sociais Transnacionais E Reação Interestatal: considerações teóricas à luz do Fórum Social Mundial e da cooperação securitária na União Européia. Caderno CRH, 19(48), 461-477.

De Souza Prais, J. L., \& da Rosa, V. F. (2017). Nuvem de palavras e mapa conceitual: estratégias e recursos tecnológicos na prática pedagógica. Nuances: estudos sobre Educação, 28(1), 201-219.

Edileusa, B., Nascimento, M., Avila, M., Batista, A. M., Silveira, A., \& De Sá, J. (2016). Relatório Final do Trabalho da Comissão Interna: Pauta Interna de Reivindicações-Categoria-Servidores Técnicos.

Goss, K. P., \& Prudencio, K. (2004). O conceito de movimentos sociais revisitado. Em Tese, 1(2), 75-91.

Herold, L. D. S. (2019). Previsão de vendas com machine learning: implementação do algoritmo prophet em linguagem R.

Jesus, J. G. D. (2013). Psicologia das massas: contexto e desafios brasileiros.

Lacerda, D. A. D. A. (2017). A atuação dos comunistas no movimento estudantil de Pernambuco: da Frente do Recife ao AI-5 (1956-1968) (Master's thesis, Universidade Federal de Pernambuco).

Larrañaga Uriarte, A. M. (2008). Análise do padrão comportamental de pedestres.

Le Bon, G. Psicologia das massas. Teodoro, 2013. (Original publicado em 1895).

Logen, M. T. Um modelo comportamental para o estudo do perfil do empreendedor. Florianópolis, 1997 (Doctoral dissertation, Dissertação (Mestrado PPEGP) Universidade Federal de Santa Catarina).

Machado, A. P., Ferreira, R., Bittencourt, I. I., Elias, E., Brito, P., \& Costa, E. (2010). Mineração de texto em Redes Sociais aplicada à Educação a Distância.Colabor@-A Revista DigitaldaCVA-RICESU,6(23).

Marques, E. (2013). A Espontaneidade das Manifestações. Revista Enfrentamento, (13).

Martins, G. D. A., \& Theóphilo, C. R. (2009). Metodologia da investigação cientifica. Atlas, 143-164.

Manzato, A. J., \& Santos, A. B. (2012). A elaboração de questionários na pesquisa quantitativa. Departamento de Ciência de Computação e EstatísticaIBILCE-UNESP, 1-17.

Merriam, S. B. (1998). Qualitative Research and Case Study Applications in Education. Revised and Expanded from" Case Study Research in Education.". Jossey-Bass Publishers, 350 Sansome St, San Francisco, CA 94104.

Nunes, C. (2014). O conceito de movimento social em debate: dos anos 60 até à atualidade. Sociologia, Problemas e Práticas, (75), $131-147$.

Paiva, A. R. (2018). Teorias do reconhecimento e sua validade heurística para a análise da cidadania e movimentos sociais no Brasil-o caso do movimento negro. Política \& Sociedade, 17(40), 258-285.

Pinto, C. R. J. (2017). A trajetória discursiva das manifestações de rua no Brasil (2013-2015). Lua Nova: Revista de Cultura e Política, 119-153.

Reisen, V. A., \& Silva, A. N. (2011). O uso da linguagem R para cálculos de estatística básica. Vitória, ES: EDUFES.

Serapião, P. R. B., Suzuki, K. M. F., \& Marques, P. M. D. A. (2010). Uso de mineração de texto como ferramenta de avaliação da qualidade informacional em laudos eletrônicos de mamografia. Radiologia Brasileira, 43, 103-107.

Tanizaka, H. (2020). Do Armário para a Cidadania: Políticas Públicas e Qualidade de Vida na População LGBTQIA+.

Tilly, C. (2010). Movimentos sociais como política. Revista brasileira de Ciência política, (3), 133-160.

Vilela, R. B., Ribeiro, A., \& Batista, N. A. (2020). Nuvem de palavras como ferramenta de análise de conteúdo. Millenium, (11), 29-36.

Wainer, J. (2007). Métodos de pesquisa quantitativa e qualitativa para a Ciência da Computação. Atualização em informática, 1(221-262), 32-33. 